\title{
ESTUDO DA SECAGEM DE PEQUI (Caryocar brasiliensis Camb.) PRECEDIDA DE DESIDRATAÇÃ̃ OSMÓTICA
}

\author{
J.L.G. CORRÊA ${ }^{1}$, F.V. FIGUEIRA ${ }^{1}$, L.F. OLIVEIRA ${ }^{2}$ e FONSECA, B.E. ${ }^{1}$ \\ ${ }^{1}$ Universidade Federal de Lavras, Departamento de Ciência dos Alimentos \\ ${ }^{2}$ Universidade Federal de São João Del-Rei, Campus Centro Oeste Dona Lindu \\ E-mail para contato: jefferson@dca.ufla.br
}

\begin{abstract}
RESUMO - O pequi é uma fruta típica do cerrado brasileiro de grande importância nutricional, gastronômica e econômica para a região. Sua utilização não é generalizada em território nacional devido, sobretudo, à sua alta perecibilidade. O objetivo deste estudo foi avaliar a secagem da polpa do pequi por dois métodos: secagem convectiva natural e secagem a vácuo, precedidas ou não de desidratação osmótica (DO) em solução de sacarose ( $40{ }^{\circ}$ Brix, $25{ }^{\circ} \mathrm{C}$ e tempo de imersão de $120 \mathrm{~min}$ ). As amostras frescas e osmoticamente desidratadas apresentaram teor de água inicial de 51,49\% e $31,86 \%$ (b.u.), respectivamente e foram secas até $13 \%$ (b.u.). Os experimentos de secagens foram realizados a 40 e $60^{\circ} \mathrm{C}$ com a retirada de amostras em tempos prédeterminados para a obtenção da cinética de secagem. O modelo difusivo de Fick foi testado para o ajuste da cinética de secagem. Tanto a utilização da DO quanto o aumento da temperatura aumentaram a difusividade de água no fruto, com consequente diminuição do tempo final de secagem. A utilização de vácuo não alterou de forma significativa o tempo do processo. O modelo de Fick conseguiu predizer as tendências observadas experimentalmente.
\end{abstract}

\section{INTRODUÇÃO}

$\mathrm{O}$ pequizeiro (Caryocar brasiliense Camb.) é uma árvore típica do cerrado brasileiro. $\mathrm{O}$ fruto desta árvore, o pequi, é economicamente explorado pela população regional que o costuma consumir em sua forma fresca, bem como o utiliza para preparo de pratos tradicionais, sucos, sorvetes, licores e geleias (Maia et al., 2008).

Além da importância regional, o pequi é também rico em antioxidantes naturais como compostos fenólicos e carotenoides. O fruto pode até mesmo ser utilizado como substituto de antioxidantes sintéticos, no enriquecimento funcional ou na suplementação alimentar. Apesar destes aspectos positivos, a alta perecibilidade do fruto torna inviável sua comercialização in natura em regiões distantes das áreas produtoras (Melo Jr et al., 2012; Machado et al., 2013). O processamento do pequi pode agregar valor ao produto brasileiro, gerar renda à população local e possibilitar a difusão de seu consumo (Machado et al., 2013; Geöcze et al., 2013).

A secagem, operação de transferência simultânea de calor e massa promove uma diminuição da atividade de água do alimento, sendo indicada como método de conservação de alimentos (de Oliveira et al., 2011; Borges et al., 2011; Fante et al., 2011; Isquierdo et al., 2013).

Dentre os métodos de secagem, o mais utilizado é a secagem convectiva, devido à facilidade de implementação com um nível tecnológico mais acessível (Jangam, 2011). Porém, a exposição prolongada de alimentos a altas temperaturas em uma secagem convectiva costuma provocar degradação de nutrientes e alteração de propriedades sensoriais como cor e textura (Cárcel et al., 
2012). Métodos de secagem menos agressivos, como a secagem a vácuo, apresentam menores alterações no produto alimentício, apesar de requerer um capital de investimento mais elevado (Aghbashlo et al., 2013; Orikasa et al., 2014).

Além do uso de métodos mais brandos de secagem, o uso de pré-tratamentos, como a desidratação osmótica (DO), pode proporcionar maior conservação da qualidade do produto seco (Fernandes et al., 2008; Fernandes e Rodrigues, 2008; Corrêa et al., 2011) A DO conduz a alimentos de atividade de água intermediária, sendo assim utilizada com a função de prétratamento a outros métodos de conservação, como a secagem (Falade e Igbeka, 2007; Fernandes et al., 2008; Fernandes e Rodrigues, 2008; Corrêa et al., 2011).

O objetivo deste trabalho foi o estudo da secagem de pequi por diferentes métodos (secagem convectiva e a vácuo), precedido ou não de desidratação osmótica, com avaliação da cinética de secagem pelo modelo difusional de Fick.

\section{MATERIAL E MÉTODOS}

\subsection{Material}

Os frutos de pequi (Caryocar brasiliense Camb.) foram adquiridos congelados, maduros e na forma de caroço, ou seja, sem o exocarpo e o mesocarpo externo. Os mesmos eram provenientes do cerrado norte mineiro colhidos na safra de dezembro de 2012. Os frutos apresentavam teor de água inicial de 51,49 \% (b.u.) e atividade de água 0,986.

Após descongelamento, os frutos foram lavados em água corrente e despolpados com auxílio de faca de aço inoxidável. Foram obtidas amostras da polpa no formato de fatias de espessura de aproximadamente 3 milímetros.

A determinação da atividade de água ocorreu à temperatura de $25 \pm 2^{\circ} \mathrm{C}$ com o uso do equipamento Aqualab, modelo CX-2T (DecagonDevices Inc., Pullman, WA, EUA).

\subsection{Desidratação Osmótica (DO)}

A desidratação osmótica foi testada como pre-tratamento. As condições de DO foram estabelecidas com base em trabalhos semelhantes (Corrêa et al., 2010; Viana et al., 2014; Perussello et al., 2014) com outros frutos. Não foram encontrados trabalhos de DO de pequi.

As amostras foram inseridas em uma solução de sacarose comercial à concentração de 40 ${ }^{\circ}$ Brix e temperatura controlada em $25{ }^{\circ} \mathrm{C}$. A relação de massa entre o alimento e a solução foi mantida constante em 1/50 para que a diluição da solução durante a desidratação osmótica fosse desprezível (Viana et al., 2014). A DO foi mantida por $120 \mathrm{~min}$. Após este período, as amostras foram retiradas da solução e imersas em um banho de gelo e água destilada por aproximadamente $10 \mathrm{~s}$ para cessar a desidratação e retirar o excesso de solução presente na superfície. As amostras tiveram sua umidade superficial seca cuidadosamente com papel absorvente e pesadas.

\subsection{Secagem}

Os experimentos de secagem de amostras in natura e frescas e desidratadas osmoticamente foram realizados a 40 e $60^{\circ} \mathrm{C}$ em estufa de convecção natural e estufa à vácuo Nos experimentos em que se utilizou vácuo, a pressão foi de $600 \mathrm{mmHg}$. Observa-se que as condições ambientais do 
período em que foram realizados os experimentos foram temperatura de bulbo seco na faixa de $25 \pm 2{ }^{\circ} \mathrm{C}$ e umidade relativa no intervalo de $55 \pm 5 \%$.

As temperaturas utilizadas neste trabalho são recorrentes em publicações de secagem convectiva (Zlatanović et al., 2013; Aghbashlo et al. 2013; Argyropoulos e Müller, 2014) e de secagem à vácuo (Orikasa et al., 2014; Aghbashlo et al. 2013). São temperaturas que não imprimem degradação significativa dos nutrientes de alimentos, bem como interferência na cor e textura devido à ação do calor.

\subsection{Cinética de secagem}

O teor de água foi monitorado ao longo do tempo no processo com retirada de amostras em tempos pré determinados até que se atingisse teor de água de $13 \%$. Para todas as condições testadas os valores de razão de teor de água foram calculados, Equação 1, e ajustados para descrever a cinética de secagem da polpa do pequi, e gráficos de razão de teor de água em função do tempo foram gerados.

$$
\mathrm{M}_{\mathrm{r}}=\frac{\mathrm{X}_{\mathrm{t}}-\mathrm{X}_{\mathrm{eq}}}{\mathrm{X}_{\mathrm{i}}-\mathrm{X}_{\mathrm{eq}}}
$$

onde $\mathrm{M}_{\mathrm{r}}=$ razão do teor de água, adimensional, $\mathrm{X}_{\mathrm{t}}=$ teor de água no tempo (b. u.) $\left[\mathrm{kg} \mathrm{kg}^{-1}\right], \mathrm{X}_{\mathrm{i}}=$ teor de água para $\mathrm{t}=0$ (b. u.) $\left[\mathrm{kg} \mathrm{kg}^{-1}\right], \mathrm{X}_{\mathrm{eq}}=$ umidade de equilíbrio (b. u.) $\left[\mathrm{kg} \mathrm{kg}^{-1}\right]$, e t $=$ tempo de secagem [min].

O modelo fenomenológico foi o baseado na equação de Fick (Crank, 1975), Equação 2.

$$
\frac{\partial \mathrm{X}(\mathrm{t})}{\partial \mathrm{t}}=\frac{\partial}{\partial \mathrm{z}}\left(\mathrm{D}_{\mathrm{eff}} \frac{\partial \mathrm{X}(\mathrm{t})}{\partial \mathrm{z}}\right)
$$

onde $\mathrm{M}_{\mathrm{r}}=$ razão do teor de água, adimensional, $\mathrm{t}=$ tempo de secagem [min], $\mathrm{k}=$ constante de secagem $\left[\mathrm{h}^{-1}\right], \mathrm{a}=$ coeficiente do modelo, onde $\mathrm{X}(\mathrm{t})$ é o teor de água no instante $\mathrm{t}, \mathrm{D}_{\text {eff }}$ é a difusividade efetiva e $\mathrm{z}$ é uma coordenada direcional genérica.

Para ajuste do modelo difusional de Fick (Equação 2) a amostra sólida foi considerada como tendo a forma de uma placa de espessura 2L. Considerou-se uma condição de teor de água inicial uniforme, $\mathrm{X}_{(\mathrm{z}, 0)}=\mathrm{X}_{0}$.

As condições de contorno são de simetria de concentração, $\left.\frac{\partial \mathrm{X}(\mathrm{t})}{\partial \mathrm{t}}\right|_{\mathrm{z}=0}=0$, e de equilíbrio do teor de água na superfície do material, $\mathrm{X}_{(\mathrm{L}, \mathrm{t})}=\mathrm{X}_{\mathrm{eq}}$. Com consideração às condições iniciais de contorno, a equação unidirecional de Fick torna-se a Equação 3:

$$
M_{r}=\left(\frac{8}{\pi^{2}} \sum_{i=1}^{\infty} \frac{1}{(2 i+1)^{2}} \exp \left(-(2 i+1)^{2} \pi^{2} D_{\text {eff }} \frac{t}{4 L^{2}}\right)\right)
$$

onde $\mathrm{M}_{\mathrm{r}}=$ razão do teor de água, adimensional, $\mathrm{D}_{\text {eff }}=$ difusividade efetiva da água $\left[\mathrm{m}^{2} \mathrm{~s}^{-1}\right], \mathrm{i}=$ número de termos da série, $\mathrm{L}=$ comprimento característico (metade da espessura da amostra) [m], $\mathrm{t}=$ tempo de secagem $[\mathrm{s}]$.

Para o ajuste pelo modelo de Fick, foram realizadas análises de regressão não linear pelo método Gauss-Newton, utilizando-se o software STATISTICA 8.0 (StatSoft, Tulsa, USA). 
Obteve-se os seguintes parâmetros estatísticos: coeficiente de determinação $\left(\mathrm{R}^{2}\right)$ (Equação 4$)$ e desvio padrão da estimativa (SE) (Equação 5):

$$
\begin{aligned}
& \mathrm{R}^{2}=\frac{\sum_{\mathrm{i}=1}^{\mathrm{n}}(\widehat{\mathrm{Y}}-\overline{\mathrm{Y}})^{2}}{\sum_{\mathrm{i}=1}^{\mathrm{n}}(\mathrm{Y}-\overline{\mathrm{Y}})^{2}} \\
& \mathrm{SE}=\sqrt{\frac{\sum_{\mathrm{i}=1}^{\mathrm{n}}(\mathrm{Y}-\widehat{\mathrm{Y}})^{2}}{\mathrm{n}}}
\end{aligned}
$$

Onde $\mathrm{R}^{2}=$ coeficiente de determinação (decimal), $\mathrm{SE}=$ desvio padrão da estimativa (decimal), $\mathrm{Y}=$ valor observado experimentalmente, $\hat{\mathrm{Y}}=$ valor calculado pelo modelo, $\overline{\mathrm{Y}}=$ média de todos os valores observados experimentalmente, $\mathrm{n}=$ número de observações.

\section{RESULTADOS E DISCUSSÃO}

\subsection{Cinética de secagem}

A Figura 1 apresenta as curvas experimentais e ajustadas pelo modelo de Fick de cinética de secagem de pequi in natura, tanto em secagem por convecção natural como por secagem à vácuo.
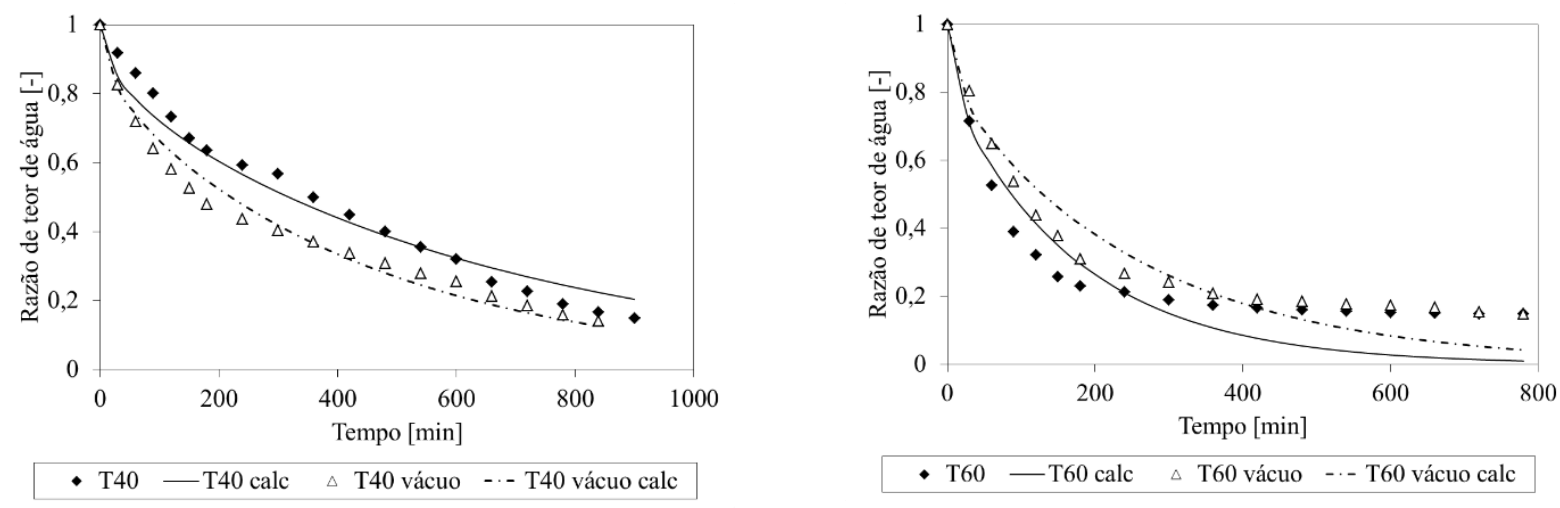

Figura 1 - Cinética de secagem de pedaços de polpa de pequi a 40 e $60^{\circ} \mathrm{C}$. calc: Valores calculados (modelo de Fick).

Ao se comparar os tempos de secagem obtidos na Figura 1 e também apresentados na Tabela 1, observa-se que houve redução no tempo de secagem e aumento da difusividade ao se trabalhar com a maior temperatura. Em processos de secagem convectiva, o aumento da temperatura leva a um aumento das taxas de transferência de energia e de massa e o tempo de secagem é, geralmente, menor (Borges et al., 2010; Borges et al., 2011).

$\mathrm{Na}$ secagem a vácuo, devido à redução do ponto de ebulição da água pela redução da pressão ambiente, há a tendência de se obter menor tempo de secagem com relação ao processo sem uso de vácuo. Porém, pela observação da Figura 1, tanto para a temperatura de $40^{\circ} \mathrm{C}$ quanto para a temperatura de $60^{\circ} \mathrm{C}$ os valores de razão de teor de água, fixado um determinado tempo, da secagem convectiva natural e da secagem a vácuo são bastante próximos. Apesar de pequena, foi observada diminuição do tempo de secagem quando se trabalhou à $40^{\circ} \mathrm{C}$. O mesmo não foi observado à $60^{\circ} \mathrm{C}$. Considera-se que a redução da resistência de secagem é mais significativo no processo de secagem quando se trabalha à $40^{\circ} \mathrm{C}$. Em temperaturas mais elevadas, a diminuição de resistência à secagem pela energia fornecida ao alimento pelo ar circundante é bem mais relevante que o eventual uso de vácuo. De maneira geral, com relação a este trabalho, considera-se que, 
dada a proximidade dos valores de tempo de secagem convectiva natural e da secagem a vácuo para ambas as temperaturas, a utilização do vácuo não produziu influência relevante em termos de redução do tempo final da desidratação.

A Figura 2 apresentam as curvas de cinética de secagem de pequi obtidas ao se utilizar o pré-tratamento osmótico.
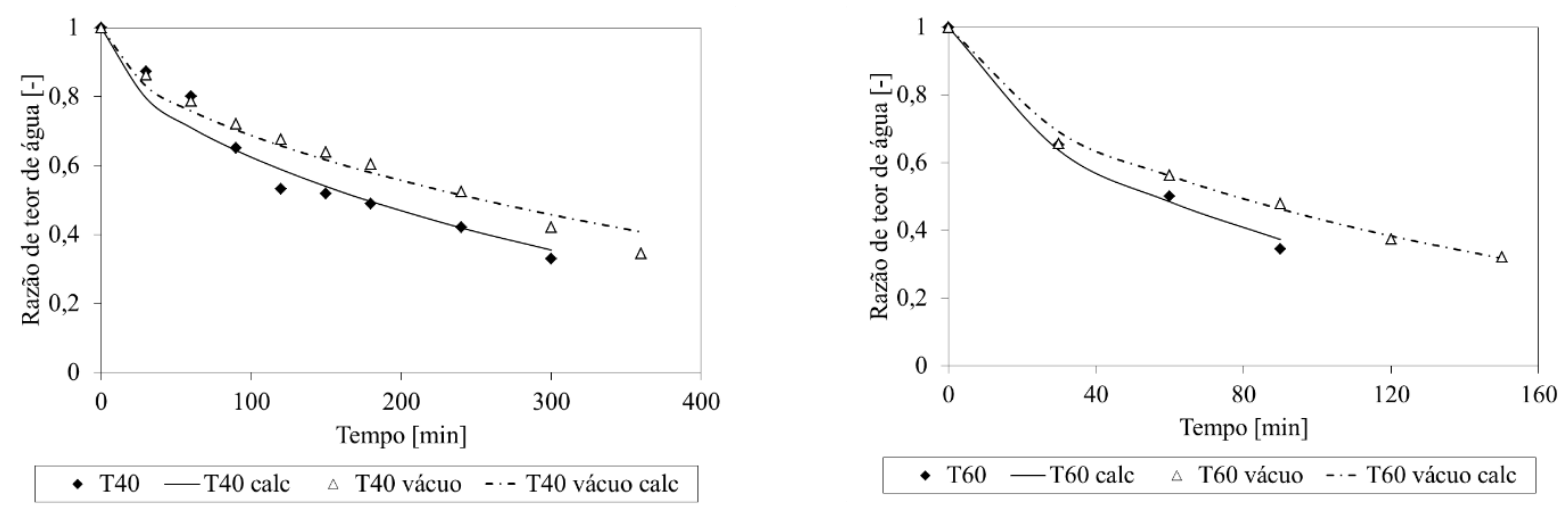

Figura 2 - Cinética de secagem de pedaços de polpa de pequi após DO a 40 e $60{ }^{\circ} \mathrm{C}$ calc: Valores calculados (modelo de Fick).

Tabela 1 - Tempos finais de secagem e dados de ajuste do modelo de Fick

\begin{tabular}{|c|c|c|c|c|}
\hline \multirow[t]{2}{*}{ Secagem } & \multicolumn{4}{|c|}{ Temperatura $40^{\circ} \mathrm{C}$, sem DO } \\
\hline & Tempo (min) & $D_{\text {eff }}\left(m^{2} s^{-1}\right)^{*}$ & $\mathrm{R}^{2}$ & $\mathrm{SE}$ \\
\hline Convectiva & 900 & $2,34 \times 10^{-11}$ & 0,9885 & 0,0490 \\
\hline \multirow[t]{2}{*}{ A vácuo } & 840 & $3,38 \times 10^{-11}$ & 0,9860 & 0,0372 \\
\hline & \multicolumn{4}{|c|}{ Temperatura $40^{\circ} \mathrm{C}$, com DO } \\
\hline Convectiva & 300 & $4,19 \times 10^{-11}$ & 0,9636 & 0,0244 \\
\hline \multirow[t]{2}{*}{ A vácuo } & 360 & $2,92 \times 10^{-11}$ & 0,9835 & 0,0160 \\
\hline & \multicolumn{4}{|c|}{ Temperatura $60^{\circ} \mathrm{C}$, sem DO } \\
\hline Convectiva & 780 & $8,56 \times 10^{-11}$ & 0,9238 & 0,1036 \\
\hline \multirow[t]{2}{*}{ A vácuo } & 780 & $5,75 \times 10^{-11}$ & 0,9456 & 0,0773 \\
\hline & \multicolumn{4}{|c|}{ Temperatura $60^{\circ} \mathrm{C}$, com DO } \\
\hline Convectiva & 90 & $1,31 \times 10^{-10}$ & 0,9947 & 0,0125 \\
\hline A vácuo & 150 & $9,52 \times 10^{-11}$ & 0,9953 & 0,0090 \\
\hline
\end{tabular}

DO significa desidratação osmótica.

De maneira geral, a DO conduziu a um aumento considerável da difusividade com redução também considerável do tempo de secagem (Figuras 1 e 2 e Tabela 1). Ao se considerar que a exposição à temperatura por tempos prolongados levam a degradação de nutrientes e de cor, o tratamento osmótico deve favorecer consideravelmente estas propriedades. O pré-tratamento osmótico foi responsável pela redução do teor de água de 51,49\% para 31,86 \% (b.u.). Apesar das amostras iniciarem os experimentos de secagem com teor de água reduzido, pode-se observar que, 
em geral, o tratamento osmótico levou a aumento da difusividade da água o que confirma que a taxa de secagem é maior devido ao pré-tratamento, independentemente da umidade inicial. A retirada de água e ingresso de sólidos na estrutura do material em uma DO traz como consequência danos na estrutura do alimento que promovem a retirada de água em uma posterior operação de secagem (Corrêa et al., 2011; Seguí et al, 2012).

Com relação à temperatura, observa-se (Tabela 1, Figura 2) que houve redução no tempo de secagem e aumento da difusividade ao se trabalhar com a maior temperatura. Como mencionado acima, o aumento da temperatura leva a um aumento das taxas de transferência de energia e de massa. Observa-se que o uso de desidratação osmótica aliado ao uso de maiores temperaturas aumentou a diferença entre os tempos de secagem com relação aos experimentos realizados sem DO (Figura1).

Com o uso de $\mathrm{DO}$, a secagem à vácuo tanto à $40^{\circ} \mathrm{C}$ quanto para à $60^{\circ} \mathrm{C}$, foi realizada com tempo de secagem e redução da difusividade com relação aos experimentos de secagem convectiva. Em trabalho semelhante de secagem de cubos de maça e batata, (Rahnan e Mujumdar, 2007) observaram que a os solutos adicionais da DO no interior do alimento interagem com a água e dificultam a retirada da mesma em uma secagem à vácuo. Apesar deste fato, a DO conduziu à redução do tempo de secagem com relação aos experimentos sem DO.

$\mathrm{Na}$ Tabela 1 além dos tempos finais de cada tratamento de secagem os dados de ajuste do modelo. Embora o modelo de Fick não tenha ajustado suficientemente bem os dados experimentais, o modelo conseguiu predizer as diferenças experimentais observadas nas diversas condições estudadas.

\section{CONCLUSÃO}

As cinéticas de secagem obtidas neste trabalho e as difusividades obtidas pelo modelo de Fick expuseram que, tanto a secagem convectiva natural quanto a secagem a vácuo possuem taxas de secagem semelhantes para a polpa de pequi in natura.

A temperatura influenciou de maneira inversa o tempo de secagem, sendo que quanto maior a primeira, menor é o segundo.

A utilização de pré-tratamento osmótico a $40^{\circ}$ Brix de sacarose reduziu consideravelmente o tempo de secagem, principalmente quando combinado a temperaturas mais altas.

A modelagem matemática da secagem pelo modelo de Fick apesar de não ajustar adequadamente os dados experimentais, serviu para quantificar as tendências observadas experimentalmente.

\section{REFERÊNCIAS}

AGHBASHLO, M.; MOBLI, H.; RAFIEE, S.; MADADLOU, A. A review on exergy analysis of drying processes and systems. Renew. Sust. Energ. Rev., v. 22, p. 1-22, 2013.

ARGYROPOULOS, D.; MÜLLER, J. Changes of essential oil content and composition during convective drying of lemon balm (Melissa officinalis L.). Ind. Crop Prod, v. 52, p. 118-124, 2014.

BORGES, S. V.; MANCINI, M. C.; CORRÊA, J. L. G.; LEITE, J. B. Drying kinetics of bananas by natural convection: Influence of temperature, shape, blanching and cultivar. Cienc. Agrot., v. 35, p. 368-376, 2011.

BORGES, S. V.; MANCINI, C.; LUIZ, J.; CORR, G.; LEITE, J. Drying of banana prata and banana d 'água by forced convection. Cie. Tecnol. de Alimentos, v. 30, p. 605-612, 2010. 
CÁRCEL, J. A.; BENEDITO, J.; \& MULET, A. Food process innovation through new technologies : Use of ultrasound. J. Food Eng., v. 110, p. 200-207, 2012.

CORRÊA, J. L. G. .; DEV, S. R. S.; GARIEPY, Y. .; RAGHAVAN, G. S. V. Drying of Pineapple by Microwave-Vacuum with Osmotic Pretreatment. Dry. Technol., v. 29, p. 1556-1561, 2011.

CORRÊA, J. L. G.; PEREIRA, L. M.; VIEIRA, G.; HUBINGER, M. D. Mass transfer kinetics of pulsed vacuum osmotic dehydration of guavas. J. Food Eng., v. 96, p. 498-504, 2010.

CRANK, J. The Mathematics of Diffusion. O. Carendon press, Ed. 2nd ed., 1975.

DE OLIVEIRA, L. F.; CORREA, J. L. G.; TOSATO, P. G.; BORGES, S. V.; ALVES, J. G. L. F.; FONSECA, B. E. Sugarcane Bagasse Drying in a Cyclone: Influence of Device Geometry and Operational Parameters. Dry. Technol., v. 29, p. 946-952, 2011.

FALADE, K. O.; IGBEKA, J. C. Osmotic Dehydration of Tropical Fruits and Vegetables. Food Rev. Int., v. 23, p. 373-405, 2007.

FANTE, C.; CORREAA, J.; NATIVIDADE, M.; LIMA, J.; LIMA, L. Drying of plums (Prunus sp, c.v Gulfblaze) treated with $\mathrm{KCl}$ in the field and subjected to pulsed vacuum osmotic dehydration. Int. J. Food Sci. Tech., v. 46, p. 1080-1085, 2011.

FERNANDES, F. A. N.; GALLÃO, M. I.; RODRIGUES, S. Effect of osmotic dehydration and ultrasound pre-treatment on cell structure: Melon dehydration. LWT - Food Sci. Tech., v. 41, p. 604-610, 2008.

FERNANDES, F. A. N.; RODRIGUES, S. Application of Ultrasound and Ultrasound-Assisted Osmotic Dehydration in Drying of Fruits. Dry. Technol., v. 26, p. 1509-1516, 2008.

GEÖCZE, K. C.; BARBOSA, L. C. A.; FIDÊNCIO, P. H., SILVÉRIO, F. O.; LIMA,C. F.; BARBOSA, M. C. A.; ISMAIL, F. M. D. Essential oils from pequi fruits from the Brazilian Cerrado ecosystem. Food Res. Int., v. 54, p. 1-8, 2013.

ISQUIERDO, E. P.; BORÉM, F. M.; ANDRADE, E. T. DE; CORRÊA, J.; OLIVEIRA, P. D. DE; ALVES, G. E. Drying kinetics and quality of natural coffee. Transactions of the ASABE, v. 56, p. 1003-1010, 2013.

JANGAM, S. V. An Overview of Recent Developments and Some R\&D Challenges Related to Drying of Foods. Dry. Technol., v. 29, p. 1343-1357, 2011.

MACHADO, M. T. C.; MELLO, B. C. B. S.; HUBINGER, M. D. Study of alcoholic and aqueous extraction of pequi (Caryocar brasiliense Camb.) natural antioxidants and extracts concentration by nanofiltration. J. Food Eng., v. 117, p. 450-457, 2013.

MAIA, J. G. S.; ANDRADE, E. H. A.; SILVA, M. H. L. Aroma volatiles of pequi fruit (Caryocar brasiliense Camb.). J. Food Comp. Analysis, v. 21, p. 574-576, 2008.

MELO JUNIOR, A. F.; CARVALHO, D.; VIEIRA, F. A.; OLIVEIRA, D. A. Spatial genetic structure in natural populations of Caryocar brasiliense Camb. (Caryocareceae) in the North of Minas Gerais, BraziL. Biochem. Syst. and Ecol., v. 43, p. 205-209, 2012.

ORIKASA, T.; KOIDE, S.; OKAMOTO, S.; IMAIZUMI, T.; MURAMATSU, Y.; TAKEDA, J.; SHIINA, T.; TAGAWA, A. Impacts of hot air and vacuum drying on the quality attributes of kiwifruit slices. J. of Food Eng., v. 125, p. 51-58, 2014.

PERUSSELlO, C. A.; KUMAR, C.; DE CASTILHOS, F.; KARIM, M. A. Heat and mass transfer modeling of the osmo-convective drying of yacon roots (Smallanthus sonchifolius). Appl. Therm. Eng., v. 63, p. 23-32, 2014. 
RAHNAN, S. M. A.; MUJUMDAR, A. S. Effect of osmotic treatment with concentrated sugar and salt solutions on kinetics and color in vacuum contact drying. J. Food Process. Pres., v. 31, p. 671-687, 2007.

SEGUÍ, L.; FITO, P. J.; FITO, P. Understanding osmotic dehydration of tissue structured foods by means of a cellular approach. J. Food Eng., v. 110, p. 240-247, 2012.

VIANA, A. D., CORRÊA, J. L. G., \& JUSTUS, A. Optimization of the pulsed vacuum osmotic dehydration of cladodes of fodder palm. Int. J. of Food Sci. Tech., v. 49, p. 726-732, 2014.

ZLATANOVIĆ; I.; KOMATINA, M.; ANTONIJEVIĆ, D. Low-temperature convective drying of apple cubes. Appl. Therm. Eng., v. 53, p. 114-123, 2013. 